LIVER

\title{
Survey of hepatitis B surface variant infection in children 15 years after a nationwide vaccination programme in Taiwan
}

\author{
H-Y Hsu, M-H Chang, Y-H Ni, H-L Chen
}

Gut 2004;53:1499-1503. doi: 10.1136/gut.2003.034223

See end of article for authors' affiliations

Correspondence to: Dr M-H Chang, Department of Paediatrics, National Taiwan University Hospital, No 7, ChungShan South Rd, Taipei, Taiwan; mhchang@

ha.mc.ntu.edu.tw

Revised version received 26 March 2004

Accepted for publication 31 March 2004
Background: It is not known whether hepatitis B virus (HBV) with mutations in the a determinant (amino acids (aa) 121-149) of the hepatitis B surface antigen (HBsAg) affect vaccination efficacy.

Aim: To investigate the prevalence and clinical significance of these mutants in children, 15 years after universal vaccination in Taiwan.

Methods: Nucleotide sequences encoding the a determinant region (aa 110-160) of HBsAg were analysed in all HBV-DNA positive sera from 1357 children and 219 adolescents serosurveyed in 1999. We then compared the prevalence and changes in the mutants in these children with our previous surveys in the same area conducted in 1984 (just before vaccination), 1989, and 1994.

Results: The prevalence of $a$ determinant mutants in HBV-DNA positive children was $7.8 \%(8 / 103)$ in 1984 , which significantly increased to $19.6 \%(10 / 51)$ in 1989 , peaked at $28.1 \%(9 / 32)$ in 1994 , and remained at 23.1\% ((3/13) (T131I, G145R, G145R)) in 1999; it was higher in those fully vaccinated compared with those not vaccinated $(15 / 46$ v 15/153; $p<0.001)$. However, the number of mutant infected children in each survey was stable in the first 5-10 year period but decreased 10-15 years post vaccination. Increased amino acid variation in the a determinant region occurred in carrier children in the post vaccination survey. Mutated residues tended to occur more frequently in the region with greater local hydrophilicity (residues 140-149) in those vaccinated than in unvaccinated children with variant infection (12/15 v 6/15; $p=0.062)$. More HBsAg positive a determinant mutants emerged in children fully vaccinated with plasma derived vaccine than those given recombinant vaccine $(10 / 2399(0.46 \%) v$ $0 / 503 ; p=0.122$ ).

Conclusion: We found that a determinant variants have an advantage in infecting immunised children but do not threaten current HBV vaccination strategies in Taiwan.
$\mathrm{S}$ ince effective vaccines against hepatitis B became available in 1982, their widespread use in many areas of the world has dramatically reduced the carrier rate of hepatitis B virus (HBV) and significantly decreased the incidence of childhood hepatocellular carcinoma. ${ }^{1-4}$ Hepatitis B surface antigen (HBsAg), used in current vaccines, contains an $a$ determinant which is located between amino acids (aa) 121-149 of HBsAg and is believed to be the major immune target of polyclonal antibody against HBsAg (antibody to hepatitis B surface antigen (anti-HBs)). The emergence of surface gene mutants, with mutations mainly occurring within the a determinant, has been observed in some vaccinees in several areas of the world..$^{5-7}$ The mutants may go undetectable by current diagnostic assays and potentially cause breakthrough infections in a vaccinated population ${ }^{7}$ thus posing a potential threat to the long term success of vaccination programmes. We have previously reported an increase in the prevalence of $a$ determinant mutants in HBVDNA positive children over a 10 year period after the introduction of universal vaccination against hepatitis $B$ in Taiwan, from $7.8 \%$ in 1984 (just before vaccination) to $19.6 \%$ in 1989 and $28.1 \%$ in $1994 .^{8}$ The prevalence of the HBsAg mutants was also significantly higher among those fully vaccinated than among those not vaccinated. ${ }^{8}$ In 1999 , 15 years after universal hepatitis B immunisation was implemented in Taiwan, we conducted a HBV seroepidemiological survey in the same district ${ }^{9}$ and found that the prevalence of chronic HBV infection among children younger than 15 years of age declined from $10 \%$ in 1984 to $0.7 \%$ in 1999. ${ }^{9}$

The child population surveyed in 1999 in Taiwan has been fully covered by nationwide vaccination programmes. The pressure on HBV to select some surface variants that can replicate in such populations increased. Understanding how common these surface mutants are in this newly surveyed population may be relevant to future vaccine design. To assess current changes in HBV surface variants in relation to universal vaccination, we analysed the prevalence and implication of $a$ determinant variants in the Taiwanese child population serosurveyed in 1999 with the highest vaccine coverage level and compared the results with our three previous surveys in children, conducted when vaccination was unavailable or only low or moderate vaccine coverage levels were achieved.

\section{SUBJECTS AND METHODS}

\section{Study subjects}

A nationwide vaccination programme against hepatitis B was conducted in 1984 in Taiwan to immunise infants of HBsAg carrier mothers. ${ }^{2}$ The programme was extended to all newborn infants and all children of preschool age in 1987 and then to schoolchildren, teenagers, and finally to adults in 1989-1990. Three successive HBV seroepidemiological studies were performed in the same areas, Chung-Cheng District, Taipei City, in 1984 (prior to the vaccination programme), in 1989 , and in $1994 . .^{10-12}$ In 1999, 15 years after the start of the vaccination programme, a similar serosurvey was conducted in the same district. Methods of recruitment and participation rates were similar to the three previous surveys. ${ }^{10-12}$

Abbreviations: aa, amino acids; $\mathrm{HBV}$, hepatitis $B$ virus; $\mathrm{HBsAg}$, hepatitis $B$ surface antigen; anti-HBs, antibody to hepatitis $B$ surface antigen; $\mathrm{HBeAg}$, hepatitis $B$ e antigen; anti-HBe, antibody to hepatitis $B$ e antigen; anti-HBc, antibody to hepatitis $\mathrm{B}$ core antigen 
Table 1 Prevalence of a determinant mutants in serum samples from children less than 15 years of age enrolled in the seroepidemiological surveys in 1984, 1989, 1994, and 1999

\begin{tabular}{|c|c|c|c|c|}
\hline & 1984 survey & 1989 survey & 1994 survey & 1999 survey \\
\hline No of children surveyed for HBV markers $†$ & 1200 & 1134 & 1515 & 1357 \\
\hline $\begin{array}{l}\text { No of children analysed for serum } \\
\text { HBV-DNA }\end{array}$ & 148 & 91 & 65 & 71 \\
\hline $\begin{array}{l}\text { No of children seropositive for } \\
\text { HBV-DNA }\end{array}$ & $103^{* * * * * *}$ & $51^{* * * * *}$ & $32^{* * * * * * *}$ & $13^{* * * * * * * * * * * *}$ \\
\hline $\begin{array}{l}\text { No of children who harboured a } \\
\text { determinant mutant } \ddagger\end{array}$ & $8(7.8 \%)^{* * * * * *}$ & $10(19.6 \%)^{* * * * *}$ & $9(28.1 \%)^{* * * * * * *}$ & $3(23.1 \%)^{* * * * * * * * * * * *}$ \\
\hline Various a determinant detected (No) & $\begin{array}{l}\text { T126A (1), M133L(1) } \\
\text { F134L(1), C138S(1) } \\
\text { T140R(1), T140I(1) } \\
\text { T143M(1), D144A(1) }\end{array}$ & $\begin{array}{l}\text { T126A(2),P127T(1) } \\
\text { Q129H(1), } \\
\text { S143W(mix)(2), } \\
\text { G145R(mix)(1) G145R(2), } \\
\text { W156L(1) }\end{array}$ & $\begin{array}{l}\text { T125A(1), P120Q+P127T(1) } \\
\text { T126A+T143M (1) } \\
\text { T126S(mix)+D144H (mix)(1) } \\
\text { D144H(mix)+G145R(1) } \\
\text { T140P(mix)(1) N146S(1), } \\
\text { T148I(1) } \\
\text { C147R(mix)+C149R(mix)(1) }\end{array}$ & $\mathrm{T} 1311(1) \mathrm{G} 145 \mathrm{R}(2)$ \\
\hline
\end{tabular}

TThe methods used for selection of children for hepatitis B virus (HBV)-DNA testing from surveyed children and various a determinant variants detected in 1984 1989 , and 1994 have been described by Hsu and colleagues ${ }^{8}$ and those in 1999 are described in the text.

‡Data in parentheses are percentage of children with a determinant mutants in HBV-DNA positive children in the survey.

${ }^{*} p=0.059,{ }^{* *} p=0.0064,{ }^{* * *} p=0.203,{ }^{* * *} p=0.91,{ }^{* * * *} p=0.98$ by $\chi^{2}$ test with Yates' correction.

Participants consisted of: (1) 157 children less than three years of age enrolled from the well baby clinic of the National Taiwan University Hospital Department of Pediatrics and from two day care centres; (2) 232 children, 3-6 years of age, recruited from two kindergarten classes; (3) 763 children, 712 years of age, enrolled from one public elementary school; (4) 205 children and adolescents, 12-15 years of age, enrolled from one public junior high school; and (5) 219 adolescents, 15-17 years of age, from a senior high school who were born before the universal vaccination programme. We defined the vaccination coverage rate as the percentage of children receiving at least three doses of HBV vaccine. Then, the total vaccination rate in the 1999 survey was $84 \%(1140 / 1357)$ in children $(<15$ years of age) and 49\% (107/219) in adolescents ( $15-17$ years of age).

\section{Detection of HBV-DNA and nucleotide sequence analysis}

After assaying for serum HBV markers in each participant using radioimmunoassays (Abbott Lab, North Chicago, Illinois, USA), sera were stored at $-70^{\circ} \mathrm{C}$ until analysed. To identify surface antigen variants in serum, all HBsAg positive sera $(n=25)$ found during this survey were included in the analysis. For those sera negative for HBsAg but positive for HBV-DNA, sera with the following specific serological profiles were also included: "antibody to hepatitis B core antigen (anti-HBc) positive only" $(\mathrm{n}=11)$ and "both antiHBC and anti-HBs positive only" $(n=51)$. HBV-DNA extracted from serum was amplified by polymerase chain reaction followed by direct sequencing of the nucleotide sequences encoding the a determinant of HBsAg (aa 110160). Detailed procedures were those described in our previous study. ${ }^{8}$

\section{Statistics}

The $\chi^{2}$ test with Yates' correction or Poisson distribution were used for statistical comparison of mutation rates between the different groups. A p value $<0.05$ was considered significant, and a $\mathrm{p}$ value between 0.05 and 0.1 was considered as showing a trend.

\section{RESULTS}

Frequency and amino acid changes of a determinant mutants in children throughout the four study cohorts In all HBV-DNA positive children from the four surveys, the prevalence of $a$ determinant mutants was eight of $103(7.8 \%)$ in 1984 which significantly increased to 10 of $51(19.6 \%)$ in 1989 , peaked at nine of $32(28.1 \%)$ in 1994 , and remained at three of $13(23.1 \%)$ in 1999 (table 1). Combining all four study cohorts, the prevalence of the mutants was higher in fully vaccinated compared with unvaccinated (15/46 v 15/ 153; $\mathrm{p}<0.001)$ HBV-DNA positive children. Among all mutant infected children, mutations tended to occur more frequently in the region with greatest local hydrophilicity (residues 140-149) in those vaccinated than in those not vaccinated $(12 / 15 \vee 6 / 15 ; \mathrm{p}=0.062)$. The G145R mutant was

Table 2 Characteristics of the children infected with mutants and amino acid changes in the a determinant of the hepatitis B virus (HBV) in the four study cohorts

\begin{tabular}{lllll}
\hline & 1984 & 1989 & 1994 & 1999 \\
\hline No of mutant infected children & 8 & 10 & 9 & 3 \\
Sex (M/F) & $4 / 4$ & $8 / 2$ & $4 / 5$ & $2 / 1$ \\
Age (y) (mean (range)) & $6.8(1.6-10.1)$ & $6.5(1.9-12)$ & $6.3(0.7-12)$ & $6.4(0.2-9.5)$ \\
No of HBeAg (+)/No of HBeAg (-) & $5 / 3$ & $8 / 2$ & $4 / 5$ & $2 / 1$ \\
No of children fully vaccinated & 1 & 3 & 8 & 3 \\
Total No of amino acid changes & 3 & 4 & 4 & 1 \\
First loop (aa 124-137) & 4 & 5 & 6 & 2 \\
Second loop (aa 139-147) & & & & \\
Changes $\times 10^{-2} /$ amino acid & 0.20 & 0.56 & 0.89 & 0.55 \\
$\quad$ First loop & 0.43 & 1.09 & 2.08 & 1.71 \\
$\quad$ Second loop & & & \\
\hline &
\end{tabular}


Table 3 Demographic data of seven subjects who harboured a determinant mutants in the 1999 survey

\begin{tabular}{|c|c|c|c|c|c|c|c|c|}
\hline & $\begin{array}{l}\text { Case } \\
\text { No }\end{array}$ & $\begin{array}{l}\text { Age } \\
\text { (y) }\end{array}$ & Sex & $\mathrm{HBsAg} /$ anti-HBs & $\mathrm{HBeAg} /$ anti-HBe & HBIG/Vac & $\begin{array}{l}\text { Maternal } \\
\mathrm{HBsAg}\end{array}$ & Mutant \\
\hline \multirow[t]{3}{*}{ Children } & $99-1$ & 0.2 & $M$ & $-1-$ & $-1-$ & $+/ 1$ & + & T1311 \\
\hline & $99-2$ & 9.5 & $\mathrm{~F}$ & $+1-$ & $+/-$ & $+/ 3$ & + & G145R \\
\hline & $99-3$ & 9.5 & $M$ & $+/-$ & $+/-$ & $+/ 4$ & + & G145R \\
\hline \multirow[t]{4}{*}{ Adolescents } & $99-4$ & 15.1 & $M$ & $+1-$ & $+/-$ & -10 & + & M133L \\
\hline & $99-5$ & 15.9 & $M$ & $+1-$ & $+1-$ & -10 & NA & $\mathrm{T} 123 \mathrm{~A}$ \\
\hline & $99-6$ & 17.3 & $\mathrm{~F}$ & $+1-$ & $+/-$ & -10 & NA & $\mathrm{P} 127 \mathrm{~T}$ \\
\hline & $99-7$ & 17.2 & $M$ & $-/+$ & $-/+$ & $-/ 0$ & NA & N146I \\
\hline
\end{tabular}

$\mathrm{HBs} A g$, hepatitis $B$ surface antigen; anti-HBs, antibody to hepatitis $B$ surface antigen; $H B e A g$, hepatitis $B$ e antigen; anti-HBe, antibody to hepatitis $B$ e antigen; $\mathrm{HBIG} / \mathrm{Vac}$, received $\mathrm{HBIG}$ injection at birth/doses of $\mathrm{HB}$ vaccine received during infancy; Maternal $\mathrm{HBsAg}$, maternal $\mathrm{HBs} A g$ status at the patient's birth; $\mathrm{NA}$, not available.

Family members were studied for hepatitis B virus (HBV) markers and HBV-DNA in serum after the 1999 survey and revealed the following:

Case 99-3: father $\mathrm{HBsAg}(+)$, S132Y; mother $\mathrm{HBsAg}(+)$, wild-type; younger sister $\mathrm{HBsAg}(-) \mathrm{HBV}-\mathrm{DNA}(-)$.

Case 99-2: serum of her mother was resampled in 2001 and was found to be $\mathrm{HBsAg}(-)$ and HBV-DNA(-).

Case 99-1, mother HBsAg(+), HBV-DNA(+), wild-type.

always observed in all three post vaccination surveys but was not found in the 1984 survey. Table 2 shows that amino acid variation in the $a$ determinant in HBV-DNA positive children was apparently greater in the three post vaccination surveys than in the prevaccination survey (1984), especially in the second loop of the a determinant region.

\section{Mutants of a determinants in children and adolescents in the 1999 survey}

Of the studied children younger than 15 years of age, serum HBV-DNA was detectable in nine of nine (100\%) HBsAg positive children, in four of $51(7.8 \%)$ children seronegative for HBsAg but seropositive for "both anti-HBs and anti-HBc positive", and in none of 11 children seropositive for "antiHBc positive only". All of the $13 \mathrm{HBV}$-DNA positive children were of genotype B. a Determinant mutants were detected in three of 13 (23.1\%) HBV-DNA positive children: two children harboured the G145R variant and an infant harboured the T131I variant (table 3 ). The prevalence of a determinant mutants in HBV-DNA positive children was significantly higher in 1989 and 1994 than in 1984 but was not significantly different between that in 1999 and 1984 (3/13 $v 8 / 103 ; \mathrm{p}=0.203$ ).

Serum positive for HBsAg was found in 16 of 219 (7.3\%) adolescents aged 15-17 years who were not part of the universal immunisation programme at birth. HBV-DNA was seropositive in 16 of $16(100 \%)$ HBsAg positive adolescents and in one of $27(3.7 \%)$ adolescents seropositive for "both anti-HBs and anti-HBc positive only". a Determinant mutants were detected in four of these 17 (23.5\%)
HBV-DNA positive adolescents (15-17 years) which was not significantly different from that (three of $13(23.1 \%)$ ) in HBV-DNA positive children ( $<15$ years). These amino acid changes involved four positions within the $a$ determinant and included the following: P127T, T123 A, M133L, and N146I (table 3).

The frequency of a determinant mutants with amino acid changes within the second loop (region with greater local hydrophilicity - that is, aa 139-147) between mutant infected children (vaccinated) and adolescents (not vaccinated) was not different $(2 / 3 \vee 1 / 4 ; \mathrm{p}>0.05)$.

\section{Prevalence of a determinant mutants in children immunised with plasma derived or recombinant vaccine}

Taken together, all vaccinees from all three post vaccination surveys (1989, 1994, and 1999), 10 of 2389 (0.42\%) cases fully vaccinated with plasma derived vaccine and none of 503 $(0 \%)$ fully immunised with yeast derived recombinant vaccine harboured HBsAg positive a determinant mutants. Also, two of $2389(0.08 \%)$ cases immunised with plasma derived vaccine and three of $503(0.6 \%)$ cases immunised with recombinant vaccine contained HBsAg negative a determinant mutants with low levels of HBV-DNA (table 4).

\section{Role of HBIG administration in the emergence of a determinant variants}

Maternal HBsAg status was known only in 34 of 45 HBVDNA positive children (25/32 in 1994 and 9/13 in 1999). Among these 34 who were also fully immunised, seven of 17

Table 4 Prevalence of a determinant mutants in children less than 15 years who were immunised with plasma derived vaccine or recombinant vaccine in 1989, 1994, and 1999

\begin{tabular}{lccl}
\hline Type of vaccine & $\begin{array}{l}\text { No of children } \\
\text { immunised }\end{array}$ & $\begin{array}{l}\text { No of immunised } \\
\text { children infected with } \\
\text { HBsAg positive mutants }\end{array}$ & $\begin{array}{l}\text { No of immunised } \\
\text { children infected with } \\
\text { HBsAg negative mutants }\end{array}$ \\
\hline $\begin{array}{l}\text { Plasma derived vaccine } \\
1989\end{array}$ & 356 & 3 & \\
1994 & 1175 & 5 & 0 \\
1999 & 968 & 2 & 1 \\
$\quad$ Total & $2399^{*}$ & $10^{*}$ & 1 \\
Recombinant vaccine & 0 & 0 & 1 \\
1989 & 114 & 0 & 0 \\
1994 & 389 & 0 & 2 \\
1999 & $503^{*}$ & $0^{*}$ & 1 \\
Total & & 3 \\
\hline HBsAg, hepatitis B surface antigen. & & \\
${ }^{*} \mathrm{p}=0.122$ by Poisson distribution. & & \\
\hline
\end{tabular}


children of carrier mothers and five of 17 children of noncarrier mothers harboured the a determinant variants $(p>0.05)$. Because children of non-carrier mothers received vaccination but without HBIG injection at their birth, HBIG is not essential for the emergence of the variants.

\section{DISCUSSION}

The universal vaccination programme in Taiwan has markedly reduced the rate of chronic carriers and the total infection rate of HBV. ${ }^{29}$ One of the remaining challenges will be to deal with potential vaccine related events. The number of HBV-DNA positive children identified during each of our four surveys rapidly fell as a result of universal vaccination. However, the prevalence of $a$ determinant mutants in HBVDNA positive children under 15 years of age was $7.8 \%$ in 1984 which increased sharply to $19.6 \%$ in 1989 , peaked at $28.1 \%$ in 1994 , and remained at $23.1 \%$ in 1999 . As vaccination coverage expanded to the whole of childhood, increased amino acid variation in the $a$ determinant in carrier children was found in each post vaccination survey, suggesting that universal vaccination might have increased a selection pressure on the emergence of the surface mutants in relation to wild-type HBV.

We found that variants numbered eight in 1984, 10 in 1989, nine in 1994, and three in 1999 out of a study population approximately equal in size for all four study periods. $^{8-12}$ This means that in the population under investigation, there was a steady decrease in the prevalence of wild-type HBV, but no change in 1989 and 1994, and even a decrease in 1999 in the absolute number of mutant infected children. This is because older chronic HBV carriers infected with wild-type virus, particularly HBeAg positive carrier mothers in Taiwan, are still the major source for children to become infected. Most vertical transmission of wild-type virus has been effectively prevented by universal immunoprophylaxis while some variants were selected for surviving in infected infants. Reasons for the limited spread of these variants are several. Firstly, none of the family members of the three variant infected children in the 1999 survey harboured the same variant, implying low transmissibility of these variants. Secondly, vaccine induced anti-HBs is usually polyclonal in nature and its binding capacity to the $a$ determinant with only one amino acid substitution may not be totally lost. ${ }^{13}{ }^{14}$ Also, anti-HBs specificities other than antia determinant may be protective. The existing recombinant vaccines were able to protect against infection with the variant G145R in chimpanzee challenge experiments. ${ }^{15}$ Therefore, current HBV vaccines possibly have cross immunity against some types of $a$ determinant mutants. Finally, in some individuals who have a more narrowly focused immune response, any loss of the limited responsive repertoire of the viral epitope could accentuate the variant virus to evade the host immunity ${ }^{16}$ : that is, only persons incapable of generating polyclonal protective anti-HBs responses may become infected with the variants.

In the 1999 survey, the prevalence of the mutants was not different between HBV-DNA positive adolescents (born before the vaccination programme) and children. In fact, these unvaccinated carrier adolescents were infected in early childhood and had been chronically infected for much longer, favouring a natural selection of $a$ determinant mutants. ${ }^{17}$ Such variants accepted more accumulation of genetic variants in the first loop than in the second loop of the a determinant region.

In one unvaccinated adolescent (case 99-7) with occult HBV infection (HBV-DNA positive but HBsAg negative), the isoleucine residue in the immunodominant site (N146I) may have resulted in lack of recognition of the variant virus by anti-HBs used in commercial assays. In fact, $a$ determinant variants may be undetectable in monoclonal based assays but more efficiently detected by polyclonal based assays ${ }^{18}$ although HBsAg negativity may be due to a very low level of serum HBsAg.

Several surface variants coexisting with wild-type virus were found in 1994 and 1989 but not found in $1999 .{ }^{8}$ Vaccine induced anti-HBs may have selected the variant but waning of anti-HBs with time may eventually favour the viral mixture reversing back to wild-type predominance. Hence some variants may not be persistent in nature. The other possibility is that some variants did not replicate as well as the wild-type virus possibly due to mutations in other unsequenced region. Of note is that G145R was always observed in the three post vaccination surveys but not found in the prevaccination era, suggesting that it is a truly vaccine escape mutant and is the most frequent and stable mutant. ${ }^{68}$

Before 1992, the only vaccine available for children in the national programme was the plasma derived hepatitis $\mathrm{B}$ vaccine (Hevac B, containing $5 \mu \mathrm{g} \mathrm{HBsAg} /$ dose) in Taiwan. The yeast derived recombinant vaccine (Engerix-B, containing $10 \mu \mathrm{g} \mathrm{HBs} \mathrm{Ag} /$ dose) was given to infants born after 1 July 1992. The prevalence of $a$ determinant variants was apparently higher in those who received plasma derived vaccine than in those who received recombinant HBV vaccine. Theoretically, plasma derived vaccine has a more diverse and immunological HBsAg protein fraction than recombinant vaccine. However, recombinant vaccine elicits a higher antiHBs response and probably confers an extended duration of protection possibly due to a higher amount of antigen in the recombinant vaccine. $^{19} 20$ A higher titre for anti-HBs in vaccinees means a higher polyclonal $a$ determinant specific antibody which has more effective binding to some variants with loss of one epitope and thus decreases the emergence of the variant in vaccinees. ${ }^{13}$ Pre-S1 and pre-S2 of HBsAg contain neutralising epitopes that are highly antigenic and in $\mathrm{H}-2$ restricted mice have the ability to stimulate nonresponders to produce anti-HBs. ${ }^{21}$ However, inclusion of pre-S epitopes does not appear to increase the efficacy of the vaccine against escape mutants. ${ }^{22}$

Our results show that HBIG is not essential for the emergence of variants. However, HBIG administration itself does select $a$ determinant mutants in transplant recipients. ${ }^{23}$ A significantly decreased prevalence of HBeAg positivity in carrier parturients between 1993 and 2000 shown in a recent study ${ }^{24}$ may have decreased the frequency of HBIG administration for infants born to carrier mothers and in turn decreased the emergence of variants, as shown in the 1999 survey.

In summary, our study in 1999 showed that with universal vaccination in childhood, the prevalence of wild-type HBV continued to decrease while the absolute number of children with infections of a determinant mutants was stable in the 510 year period after vaccination, and eventually decreased 15 years post vaccination. G145R was always observed in post vaccination surveys, suggesting that it is the most stable mutant and needs to be incorporated into future vaccine design. Our results seem to favour the view of recent mathematic models of HBV vaccination which predicts that even the current vaccine provides no cross immunity against variants and variants will not become dominant over the wild-type for at least 50 years. ${ }^{25}$ Whether infections with some variants are transient in nature, current vaccines are cross protective against mutants, or use of recombinant vaccines produce less variants requires further study.

\section{ACKNOWLEDGEMENT}

Supported by Grant NSC 89-2314-B002-393 from the National Science Council, Taiwan. 


\section{Authors' affiliations}

H-Y Hsu, H-L Chen, Department of Paediatrics, National Taiwan University Hospital, Taipei, Taiwan, and Department of Primary Care Medicine, National Taiwan University College of Medicine, Taipei, Taiwan

M-H Chang, Y-H Ni, Department of Paediatrics, National Taiwan University Hospital and College of Medicine, Taipei, Taiwan

\section{REFERENCES}

1 Coursaget P, Yvonnet B, Chotard J, et al. Seven year study of hepatitis B vaccine efficacy in infants from an endemic area in Senegal. Lancet 1986;2:1143-5.

2 Hsu HM, Chen DS, Chuang CH, et al. Efficacy of a mass hepatitis B vaccination program in Taiwan. JAMA 1988;260:2231-5.

3 Whittle HC, Inskip H, Hall AJ, et al. Vaccination against hepatitis $B$ and protection against chronic viral carriage in the Gambia. Lancet 1991:337:747-50.

4 Chang MH, Chen $\mathrm{CJ}$, Hsu HM, et al. Universal hepatitis B vaccination in Taiwan and the incidence of hepatocellular carcinoma in children. Taiwan Childhood Hepatoma Study Group. N Engl J Med 1997;336:1855-9.

5 Carman WF, Zanetti AR, Karayianis P, et al. Vaccine-induced escape mutant of hepatitis B virus. Lancet 1990;336:325-9.

6 Hsu HY, Chang MH, Ni YH, et al. Surface gene mutants of hepatitis B virus in infants who develop acute or chronic infections despite immunoprophylaxis. Hepatology 1997;26:786-91.

7 Fortuin M, Karthigesu V, Allison L, et al. Breakthrough infections and identification of a novel variant in Gambian children immunized with hepatitis B vaccine. J Infect Dis 1994;169:1374-6.

8 Hsu HY, Chang MH, Liaw SH, et al. Changes of hepatitis B surface antigen variants in carrier children before and after universal vaccination in Taiwan. Hepatology 1999;30:1312-17.

$9 \mathrm{Ni} \mathrm{YH}$, Chang MH, Huang $L M$, et al. Hepatitis B virus infection in children and adolescents in a hyperendemic area: 15 years after mass hepatitis $B$ vaccination. Ann Intern Med 2001;135:796-80.

10 Hsu HY, Chang MH, Chen DS, et al. Baseline seroepidemiology of hepatitis B virus infection in Taipei, 1984: A study just before a mass hepatitis B vaccination program in Taiwan. J Med Virol 1986;18:301-7.

11 Tsen YJ, Chang MH, Hsu HY, et al. Seroprevalence of hepatitis B infection in children in Taipei, 1989: five years after a mass hepatitis B vaccination program. J Med Virol 1991;34:96-9.
12 Chen $\mathrm{HL}$, Chang MH, Ni YH, et al. Seroepidemiology of hepatitis B virus infection in children: Ten years after a hepatitis $B$ mass vaccination program in Taiwan. JAMA 1996;276:906-8.

13 Waters JA, O'Rourke SM, Richardson SC, et al. Qualitative analysis of the humoral immune response to the " $a$ " determinant of HBs antigen after inoculation with plasma-derived or recombinant vaccine. J Med Virol 1987;21:155-60.

14 Chiou HL, Lee TS, Kuo J, et al. Altered antigenicity of 'a' determinant variants of hepatitis B virus. J Gen Virol 1997;78:2639-45.

15 Ogata N, Cote PJ, Zanetti AR, et al. Licensed recombinant hepatitis B vaccines protect against infection with the prototype surface gene mutant of hepatitis $B$ virus. Hepatology 1999;30:779-86.

16 Chisari FV, Ferrari C. Hepatitis B. virus immunopathogenesis. Ann Rev Immunol 1995;13:29-60.

17 Yamamoto K, Horkita M, Tsuda F, et al. Naturally occurring escape mutants of hepatitis $B$ virus with various mutations in the $S$ gene in carrier children seropositive for antibody to hepatitis B surface antigen. J Virol 1994;68:2671-6

18 Ireland JH, O'Donnell B, Basuni AA, et al. Reactivity of 13 in vitro expressed hepatitis $B$ surface antigen variants in 7 commercial diagnostic assays. Hepatology 2000;31:1176-82.

19 Most J, Larcher C, Vogetseder W, et al. Recombinant versus plasma-derived hepatitis $B$ vaccine: comparison of immunogenicity in medical students. Vaccine 1992;10:740-1.

20 Cheng KF, Chang MH, Lee CY, et al. Response to supplementary vaccination with recombinant or plasma hepatitis $B$ vaccine in healthy non-responding children. Vaccine 1994;12:889-902.

21 Milich DR, McLachlan A, Chisari FV, et al. Immune response to the pre-S (1) region of the hepatitis $B$ surface antigen $(\mathrm{HBs} A g)$ : A pre-S(1)-specific T cell response can by pass nonresponsiveness to the pre-S(2) and $S$ regions of HBsAg. J Immunol 1986;137:315-22.

22 Surya IGP, Kishimoto S, Sudaryats, et al. Prevention of mother-to-infant transmission of hepatitis B virus with use of a pre-S2-containing vaccine in Bali, Indonesia. Vaccine Res 1996;5:203-13.

23 Carman WF, Trautwein C, van Deursen FJ, et al. Hepatitis B virus envelope variation after transplantation with and without hepatitis immune globulin prophylaxis. Hepatology 1996;24:489-93.

24 Lin $\mathrm{HH}$, Kao JH, Chang TC, et al. Secular trend of age-specific prevalence of hepatitis B surface and e antigenemia in pregnant women in Taiwan. J Med Virol 2003;69:466-70.

25 Wilson JN, Nokes DJ, Carman WF. The predicted pattern of emergence of vaccine-resistent hepatitis B: a cause for concern? Vaccine 1999;17:973-8.

\section{EDITOR'S QUIZ: GI SNAPSHOT}

\section{Answer}

From question on page 1493

Small bowel radiography revealed a large mass dislodging and compressing the bowel towards the inferior quadrants of the abdomen. No evidence of infiltration by the tumour was detected.

At laparotomy, a large tumour originating from the anastomotic ileal loop was resected en bloc. Surprisingly, histology showed the presence of a GIST. The tumour developed from connective tissue of the peri-anastomotic ileal loop. At this site, histological signs of Crohn's recurrence were also present.

This is the second case reported in the literature of GIST occurring in a patient with Crohn's disease. Many types of non-adenocarcinoma tumours have been reported to significantly affect patients with Crohn's disease but this occurs only in a small number of patients. However, attention should be paid to the association of small bowel tumours such as GIST and Crohn's disease due to the increased difficulty of diagnosis with non-specific symptoms. 